\title{
RF Energy Harvesting using Wireless Sensor Network for Low Power Applications
}

\author{
K. Umapathy, D. Muthukumaran, S. Chandramohan
}

\begin{abstract}
The evolution of telecommunications systems emphasizes the importance of RF energy in the surroundings. This energy can be utilized for less power device namely wireless sensor network. The performance of small DC/RF converter is calculated in this paper in sequence to enslave the WSN performance for energy recovered. Specially to attain the performance in GSM band the efficient rectifier is achieved. Based on the selection of rectifying diode the design methodology works and causes the losses in rectifying antenna. By employing the slope method the advantageous performance is obtained in Advanced Design System (ADS) software. Implementing Schottky diodes in rectifier and voltage doubler HSMS 2850 is used. RF/DC conversion the maximum efficiency is $38 \%$ is achieved with $10 \mathrm{dBm}$ input power level. A uniform distribution of sensor node with network loads is utilized to control the WSN performance of Base station location as well as distance. For such reason Low Energy Adaptive Clustering Hierarchy (LEACH) protocol is utilized.
\end{abstract}

Keywords : HSMS 2850 Diode Rectenna, RF/DC Efficiency, WSN, Leach Protocol.

\section{INTRODUCTION}

The necessitate to prevent natural disasters due to changes in the environment has paved way for the development of sensor nodes in latest years [1]. The nodes of devices are small devices which have the ability to do easy measurements in nearby environment. The function of such node is transmitting and collecting the information with the help of radio waves which forms the WSN. WSN has the advantage of offering network flexibility as well as mobility when to compare to wired network.

In addition, a wired system constuiting a number of nodes can be difficult in particular applications namely structural monitoring [2]. For instance to monitor light, people present and various environmental situations with better resolutions in same building hundreds of sensor nodes is used in IoT applications [3],[4].Moreover, the WSN development is emphasized by latest version in the small rechargeable batteries growth [5] offered to the sensor node with the life

Revised Manuscript Received on December 30, 2019.

* Correspondence Author

K. Umapathy*, Associate Professor, Department of ECE, SCSVMV, Kanchipuram, India. Email: umapathykannan@gmail.com

D. Muthukumaran, Assistant Professor, Department of ECE, SCSVMV, Kanchipuram, India. Email: sarvamkumaran@gmail.com

S. Chandramohan, Assistant Professor, Department of ECE, SCSVMV, Kanchipuram-631561.

(C) The Authors. Published by Blue Eyes Intelligence Engineering and BY-NC-ND license (http://creativecommons.org/licenses/by-nc-nd/4.0/) Sciences Publication (BEIESP). This is an open access article under the CC

time corresponding to the battery capacity. Battery is the major constraint in the WSN operations due to the function of replacing/recharging the batteries is high price during placing the WSN in hard to reach position. To overcome such problem recycling ambient energy namely the sun, vibration, wind, electromagnetic wave, light, heat placed in surrounding area of sensor point. Such study is referred as Energy harvesting [6].Converting one energy source into DC power is energy harvesting. The difference in the Energy Harvesting techniques thus arises by choice of the utilized main source. Well Known Sources namely wind, sun, and vibrations have been illustrated via different transduction mechanisms, to produce sufficient quantity of energy [6] [7] [8].Though, such sources are intermittent as they based on the variations in the sun and wind seasons. For other temperature like heat are still in mechanical vibrations [9-12]. Due to this reason, the selection is emphasized of energy and illustrates a consistency level because of number of radiation sources which is shown in the Figure 1.

Research on WSNs has been focused by a general parameter: Energy efficiency. The WSN node is generally powered by batteries. If the energy becomes drain at once, the node is "dead." The batteries can be recharged or replaced only in certain situations. Moreover the recharging/replacement operation is found to be slow ,costly, and decreases network performance. Different methods namely duty cycle and power control based process is put forth to minimize the energy .
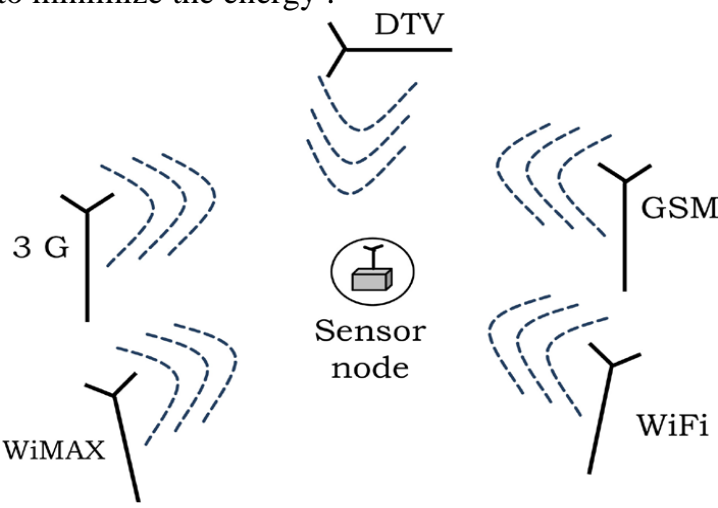

Fig. 1. Source of Radio wave radiations.

\section{PROPOSED SYSTEM OF RF/DC CONVERTER WITH HIGH-EFFICIENCY FOR AMBIENT POWER HARVESTING IN GSM BAND}


In this work, main aim is to intend the small circuit to convert the ambient RF energy with effective manner. Better power density in GSM band is considered. The smallness of above circuit includes a rectifying diode and later located in a Schenkel voltage doubler rectifier which is illustrate in the figure 2.The circuit provides a DC voltage output equivalent to double the input AC amplitude signal.

\section{A. Selection of Rectifying Diode}

It is a vital component of rectenna because shaping of RF signals received by the antenna. It is highly significant to go for high-sensitivity rectifier to provide sufficient quantity of DC power keeping in view two factors - received low power density, high frequency of signals and to reach the maximum conversion efficiency.

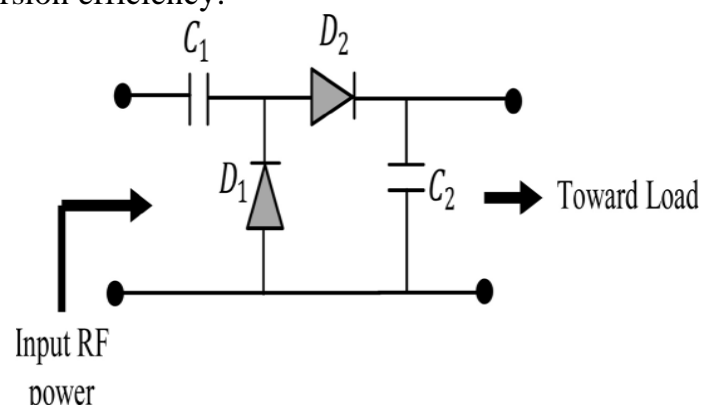

Fig. 2.Schenkel Voltage Doubler Rectifier.

\section{B. Battery models}

Generally batteries are perfect storage energy devices comprising a fixed sum of energy in units. For example a network process that involves - receiving or sending a packet, utilizes a specific amount of energy units, based on the cost of operation. The battery capacity is supposed to be minimized by process only when the process is done. The real batteries will process in various manner. Moreover all batteries will undergo self-discharge due to internal chemical activity. Additionally, when discharging and charging the battery some energy is lost. In addition, when discharging and charging battery a few energy is lost.

\section{RESULT AND DISCUSSION}

In Schenkel-type voltage doubler the selected diode HSMS 2850 is utilized. Realization and simulation of the circuit is done along with the components ranges, is illustrated in Fig. 3. In the circuit, a SMA connector connects the rectifier to the microwave source. The circuit is designed using ADS software. At $4.7 \mathrm{nF}$ the capacitor values is setted initially and the load resistance is operated $-10 \mathrm{dBm}$ input power. The outcome shows that the designed circuit can obtain a -22.5 $\mathrm{dBm}$ maximum power with an $1.23 \mathrm{k} \Omega$ optimum load.
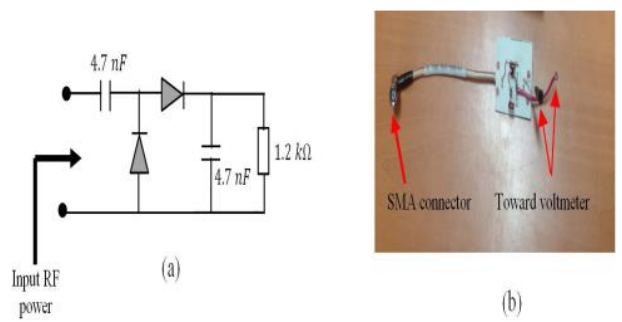

Fig. 3.Simulated and Manufactured Rectifier Circuit .

\section{CONCLUSION}

In this paper, Energy harvesting system design using WSN on the basis of recovering the ambient electromagnetic energy is presented. At first the sensor node energy budget in WSN LEACH protocol is evaluated.In GSM band to attain the good performance the small rectenna is designed in GSM band is realized. The small circuit is made up of a simple rectifier for shaping the RF signals. From the comparison of different diodes used for RF/DC conversion that Avago HSMS 2850 diode is the right choice and losses is decreased during such process of conversion. The result of the rectifier shows that an output $2.2 \mu \mathrm{W}$ DC power is dissipated with an input power level of -10dbM and an optimum load resistance of $1.2 \mathrm{k} \Omega$. The improved energy is utilized to demonstrate the physical limit on a WSN. This energy is compared with the sensor node energy budget in LEACH protocol. Distance between WSN and base station is utilized as enslavement parameter. For fast varying occurrence, Base station is placed inside or close to WSN. Though for the last round, the BS is placed away from 3kmform the WSN covered area.

\section{REFERENCES}

1. A. Mouapi, "Conception et réalisation d'une alimentation autonome pour un réseau de capteurs sans fil appliqué dans les transports ferroviaires". http://www.(Uhttp://depositum.uqat.ca/639/.

2. V. A. Kottapalli, A. S. Kiremidjian, J. P. Lynch, E. D. Carryer, T. W Kenny, K. H. Law, and Y. Lei, " Two-tiered wireless sensor network architecture for structural health monitoring", Smart Systems and Nondestructive Evaluation for Civil International Society for Optics and Photonics Infrastructures, Aug 2003,Vol.5057, pp. 8-19.

3. H, J. Visser, " Miniature rectenna design", IEEE International Applied Computational Electromagnetics Society Symposium-Italy, Mar.2017, pp. 1-2.

4. L. Atziori, A. Iera, and G. Morabito," The Internet of Things: A Survey." Computer Networks,Vol.54,No.15,oct 2010,pp.2787-2805.

5. D. Ilic, M. Kilb,K. Holl,H. W. Praas, and E. Pytlik, "Recent progress in rechargeable nickel/metal hydride and lithium-ion miniature rechargeable batteries", Journal of power sources, Vol.80,No.1-2, Jul.1999, pp.112-115.

6. S. Chalasani, and J.M Conrad, "A survey of energy harvesting sources for embedded systems". IEEE Southeast Conference, Apr.2008, pp. 442-447.

7. Y. K. Tan, and S. K. Panda, "Optimized wind energy harvesting system using resistance emulator and active rectifier for wireless sensor nodes", IEEE transactions on power electronics, Vol.26,No.1, Jul.2010, pp.38-50.

8. A. Mouapi, N. Hakem, N. Kandil, and G. V. Kamani, “ Energy harvesting design for autonomous Wireless Sensors Network applied to trains". IEEE International Ultrasonics Symposium, Sep.2016,pp. 1-4.

9. A. Mouapi, and N. Hakem, "Performance evaluation of wireless sensor node powered by RF energy harvesting". IEEE Mediterranean Microwave Symposium, Nov.2016, pp. 1-4.

10. L. M. Oliveira, and J. J. Rodrigues," Wireless Sensor Networks: A Survey on Environmental Monitoring",Vol.6,No.2, Apr.2011, pp.143-151.

11. M. Piñuela,P. D. Mitcheson, and S. Lucyszyn, "Ambient RF energy harvesting in urban and semi-urban environments", IEEE Transactions on microwave theory and techniques, Vol. 61,No.7, May 2003, pp.2715-2726.

12. T. A., Shanmugasundaram, V. Vijayabaskar, "A novel approach for energy efficient clustering in heterogeneous w ireless sensor networks", ARPN Journal of Engineering and Applied Sciences, Vol. 10, No. 5, 2015, pp. 2172-2176 


\section{AUTHORS PROFILE}

K. Umapathy, is working as Associate Professor in the Department of ECE at Sri Chandrasekharendra Saraswathi Viswa MahaVidyalaya, Kanchipuram. He obtained his UG and PG degrees in Engineering from Madurai Kamraj University and Anna University respectively. He received his $\mathrm{PhD}$ degree from JNT Univeristy, Anantapur in the year 2014. He has 24 years of experience in the field of Engineering Eduation and Adminstration. He publihsed more than 100 papers in Journals and Conferences at the level of both National and International. He authored five books in the field of Engiineering. He is a member of various professional bodies such as ISTE, IEEE, IETE etc.

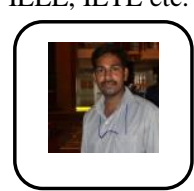

D. Muthukumaran, is working as an Assistant Professor in ECE department of SCSVMV (Deemed to be university), Kanchipuram,Tamilnadu. I have completed my bachelor degree in AMS College of engineering, Chennai and my Masters Degree in Bharath University. Currently pursuing Ph.D in SCSVMV.

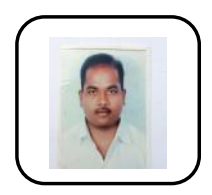

S. Chandramohan, obtained his BE degree in Electronics and Communication Engineering from ANNA University in the year 2002. He completed his M.Tech degree in Space Technology and Applications from Indian Institute of Science, Bangalore and Pursuing PhD from SCSVMV (Deemed to be University), Kanchipuram. He has nearly ' 15 ' years of Experience in the field of Engineering Education and Administration. He has published 07 papers in Journals and Conferences both at the level of National and International. He is a member of various professional bodies such as IEEE and IAENG. Currently he is working as Assistant Professor in the Department of ECE, SCSVMV (Deemed University), Kanchipuram 